\title{
Fenologia reprodutiva de espécies arbóreas em área fragmentada de Mata Atlântica em Itaborai, RJ
}

\author{
Juliana Müller Freire ${ }^{1}$, Marina Coimbra de Azevedo², Carlos Fernando Cunha1', Telmo Félix da Silva ${ }^{3}$, Alexander Silva de Resende ${ }^{1}$ \\ ${ }^{1}$ Embrapa Agrobiologia, Rodovia BR 465, km 7, CEP 23891-000, Seropédica, RJ, Brasil \\ ${ }^{2}$ Universidade Federal Rural do Rio de Janeiro, BR 465, Km 7, CEP 23890-000, Seropédica, RJ, Brasil \\ ${ }^{3}$ In memorian
}

"Autor correspondente:
juliana.muller@embrapa.br

Termos para indexação:

Fenologia

Floração

Frutificação

Sementes

\section{Index terms:}

Flowering

Fruiting

Phenology

Seeds

Histórico do artigo:

Recebido em 12/11/2013

Aprovado em 09/08/2013

Publicado em 30/09/2013

doi: 10.4336/2013.pfb.33.75.454
Resumo - Estudos fenológicos podem servir de subsídio para ações de colheita de sementes e recuperação de áreas degradadas, contribuindo para a conservação das espécies florestais. Este trabalho teve como objetivo conhecer a época de floração e frutificação de 21 espécies arbóreas nativas em área fragmentada de Mata Atlântica em Itaboraí, RJ. Foram acompanhadas mensalmente 91 matrizes, no período de janeiro a dezembro de 2011. A maior parte das espécies floresceu no final da estação seca e início da chuvosa, tendo sido observada correlação moderada e negativa entre floração e fotoperíodo. Observaram-se dois picos de frutificação: de março a abril para as espécies zoocóricas, e de setembro a outubro para espécies não zoocóricas, com correlação positiva entre precipitação e frutificação de espécies zoocóricas. Foi alta a incidência de patógenos nos frutos, comprometendo a produção de sementes de Machaerium hirtum, Melanoxylon brauna, Machaerium brasiliense e Psidium guineense. Outras espécies apresentaram baixa produção de frutos, como Andira sp., Swartzia oblata, Guarea guidonea e Enterolobium glaziovii. A sincronia de floração e frutificação entre as matrizes foi baixa para a maioria das espécies. A falta de trabalhos de fenologia para a maioria das espécies estudadas (52\%) reforça a necessidade de pesquisa para a região de estudo.

\section{Reproductive phenology of tree species in a fragmented area of Atlantic Forest, Rio de Janeiro State, Brazil}

\begin{abstract}
Phenological studies can support actions of seed collection and recovery of degraded areas, contributing to the conservation of forest species. This study aimed to understand the timing of flowering and fruiting of 21 native tree species in fragmented area of Atlantic Forest in Itaboraí, Rio de Janeiro State, Brazil. Ninety one trees were monitored monthly, from January to December of 2011. Most species flowered in the late dry season and early rainy season, and presented a moderate negative correlation between flowering and photoperiod. There were two peaks of fruiting: March-April to zoochoric species and September-October for not zoochoric species, with positive correlation between rainfall and fruiting of zoochoric species. There was a high incidence of pathogens in fruits, affecting the seed production by Machaerium hirtum, Melanoxylon brauna, Machaerium brasiliense and Psidium guineense. Other species showed low fruit production, as Andira sp., Swartzia oblata, Guarea guidonea, and Enterolobium glaziovii. The timing of flowering and fruiting among individuals was low for most species. The lack of phenology research of most species studied (52\%) reinforced the need of such studies in this region.
\end{abstract}




\section{Introdução}

A recomendação de plantio de espécies florestais nativas em áreas degradadas muitas vezes esbarra em questões práticas de obtenção de sementes, devido ao alto custo da colheita de sementes em matrizes florestais, além de empecilhos burocráticos e legais, como coleta de sementes em unidades de conservação e necessidade de registro do colhedor junto aos órgãos competentes.

Projetos de reflorestamento têm recomendado cada vez mais a coleta de sementes de ecótipos locais, amostrando um número adequado de árvores matrizes (Kageyama \& Gandara, 2000; Schimizu, 2007; Mijnsbrugge et al., 2010). Estudos têm indicado que as sementes que sejam coletadas em, no mínimo, 12 matrizes (Sebben, 2002; Piña-Rodrigues et al., 2007). Entretanto, é difícil conseguir este número de árvores para colheita de sementes num mesmo período para produção de mudas, sendo esta recomendação raramente praticada pelos viveiros florestais, que vem efetuando a colheita em cerca de cinco matrizes (Rio de Janeiro, 2010). Esta deficiência pode ocorrer devido à distribuição espacial aleatória e rara de algumas espécies, como por exemplo, Melanoxylon brauna (Carvalho et al., 2007); à assincronia na reprodução, ou seja, poucos indivíduos de uma população florindo ou frutificando num mesmo período, como já foi observado para Eugenia involucrata (Tonetto et al., 2013); ou devido à presença de pragas e doenças, reduzindo o poder germinativo das sementes.

Estudos recentes de cadastramento de árvores matrizes têm sido cada vez mais comuns, buscando aliar a conservação da base genética a projetos de recuperação de áreas degradadas (Moreira \& Anania, 2005; Medeiros \& Nogueira, 2006; Pires, 2008). Entretanto, para complementar estas ações e gerar os conhecimentos necessários para definição de estratégias de conservação e de manejo florestal é importante a realização de estudos de fenologia (Mantovani et al., 2003). A fenologia tem como base a observação de fenofases das espécies, que são os estádios de desenvolvimento, como a emergência das gemas, o desenvolvimento das folhas, a floração, a frutificação, a descoloração das folhas e a senescência. Assim, o conhecimento da floração e frutificação permite estimar períodos de reprodução das plantas, seus ciclos de crescimento, sua relação com o clima, e o melhor período para colheita de sementes (Fournier, 1974; 1976; Morellato, 1995; Mariot et al., 2003).
A elaboração de calendários fenológicos direcionados para cada região é fundamental, tendo em vista a falta de fontes bibliográficas confiáveis com este tipo de informação. A maioria das pesquisas sobre fenologia tem sido realizada em ambientes florestais sob condições climáticas sazonais. Além disso, estudos enfocando comunidades têm sido mais frequentes do que os estudos sobre uma ou poucas espécies (Bencke \& Morellato, 2002).

Considerando o contexto de forte degradação antrópica que o bioma Mata Atlântica vem sofrendo ao longo dos anos, torna-se urgente a elaboração e aumento do número de estudos que contribuam para o entendimento do processo de reprodução e regeneração das plantas nativas, e que subsidiem ações de recuperação das áreas degradadas.

O presente estudo teve como objetivo o acompanhamento fenológico de 21 espécies arbóreas, em Itaboraí, RJ, visando subsidiar a atividade de coleta de sementes para o projeto de reflorestamento que vem ocorrendo na região.

\section{Material e métodos}

\section{Área de estudo}

O estudo da fenologia foi realizado em área fragmentada de Mata Atlântica, em Itaboraí, Região Metropolitana do Rio de Janeiro, Brasil (22 $44^{\circ} 40^{\prime \prime} \mathrm{S}$ e 4251'34”W). O local de estudo abrange uma área de baixada, com altimetria variando de 0 a $400 \mathrm{~m}$, e presença de argissolo, cambissolo, latossolo ou planossolo, bastante sujeito a alagamento. A região compreende um mosaico de formações vegetais em vários níveis de degradação, onde se encontram as Florestas Ombrófilas Densas submontanas e aluviais (Lima et al., 2006). São encontrados poucos fragmentos florestais na região com tamanho variando de 1 a 100 hectares, em diferentes estágios de regeneração, mas em sua maioria em estágio precário de conservação, com baixa diversidade florística. Dentre as espécies comumente encontradas destacam-se Tabernaemontana laeta, Attalea humilis, Gochnatia polymorpa e Cecropia pachystachya.

O clima é classificado como tropical úmido ou subúmido (Aw) segundo critério de Köppen (1948). A precipitação da região varia de 1.000 a $1.500 \mathrm{~mm}$ anuais, com forte concentração de chuvas nos meses de novembro a abril (Bonnet et al., 2009). A média das 
máximas de temperatura é de $26^{\circ} \mathrm{C}$ no verão, enquanto que a média das mínimas ocorre no inverno e gira em torno de $24{ }^{\circ} \mathrm{C}$ (Bonnet et al., 2009).

Durante o ano de 2011, o total de chuvas registrado foi de $916,93 \mathrm{~mm}$, sendo dezembro e março os meses mais chuvosos. Os meses mais secos foram julho e setembro, este último de forma atípica no ano analisado: a média de precipitação do mês de setembro em outros anos é de $90,1 \mathrm{~mm}$, contrastando com 7,4 mm em setembro de 2011. Em relação à temperatura, os meses mais quentes foram janeiro e fevereiro, e os meses mais frios, junho e julho. Na Figura 1 é apresentado um climatograma para o ano de 2011.

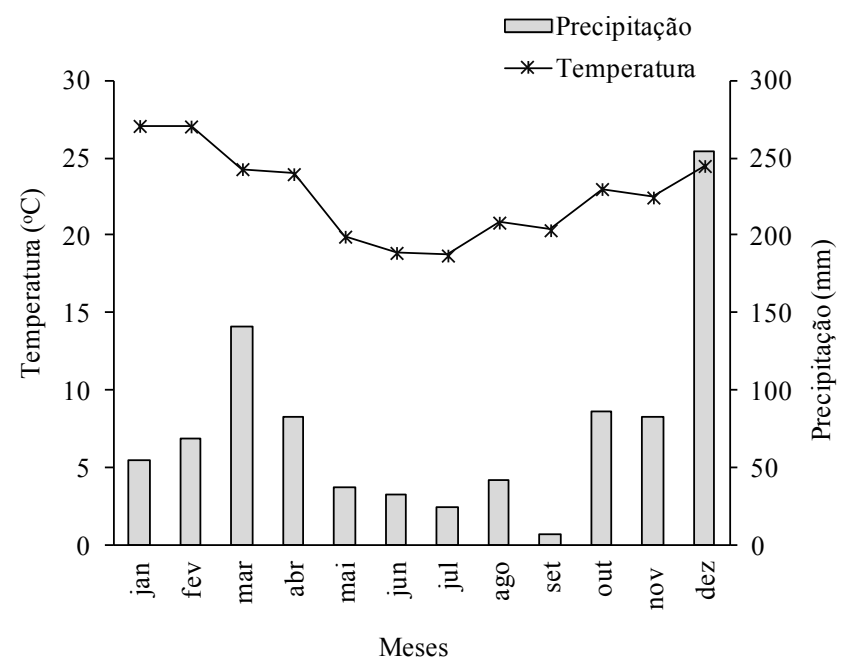

Figura 1. Distribuição da precipitação pluviométrica e das temperaturas médias mensais no período de janeiro a dezembro de 2011, na Estação de Sambaetiba, Itaboraí, RJ.

\section{Acompanhamento fenológico}

Em janeiro de 2011 foram marcadas, com placas de alumínio, 91 matrizes de 21 espécies para acompanhamento fenológico. O número de matrizes marcadas para cada espécie variou de 2 a 11. Buscou-se marcar, no mínimo, cinco matrizes para cada espécie, entretanto nem todas apresentaram densidade suficiente para atingir esta meta. Foram selecionadas espécies de ocorrência natural na região, com potencial para recuperação de áreas degradadas e/ou espécies da família leguminosae. Os critérios utilizados para selecionar as matrizes foram baseados em premissas utilizadas para seleção de árvores para coleta de sementes: (1) distância de $100 \mathrm{~m}$ entre matrizes, buscando evitar grau de parentesco entre elas; (2) indivíduos em fase adulta, avaliados a partir do tamanho (altura, DAP), variável para cada espécie, ou que já estivessem em idade reprodutiva; (3) facilidade de acesso (não foram marcadas matrizes no interior de fragmentos florestais, sempre na borda, ou em áreas de pastagens, com capoeiras), buscando facilitar a coleta futura das sementes para fins de produção de mudas.

Para cada indivíduo marcado foram registradas as coordenadas geográficas, medida a circunferência do tronco à altura do peito (CAP) e altura total, determinado o tipo de solo (hidromórfico, semi-hidromórfico, não hidromórfico), grau de isolamento e densidade. O tipo de solo foi avaliado visualmente através da observação da presença/profundidade de alagamento ao longo do ano. Considerou-se hidromórfico o solo alagado por pelo menos um mês ao ano, semi-hidromórfico, o solo úmido porém sem sinais de alagamento, e o solo não hidromórfico aquele com boa drenagem, situado principalmente em encostas. O grau de isolamento da matriz, assim como sua densidade foi avaliada pela presença ou não de outras matrizes da mesma espécie num raio de $500 \mathrm{~m}$, efetuando-se a sua contagem.

As espécies foram classificadas de acordo com o tipo de dispersão de seus frutos (anemocórica, zoocórica, barocórica, autocórica) e em grupos ecológicos de acordo com informações secundárias disponíveis na bibliografia (Carvalho, 2003, 2006). Para grupo ecológico foi utilizada a classificação de Gandolfi et al. (1995) que distingue três grupos sucessionais: pioneira, secundária inicial e secundária tardia.

O acompanhamento fenológico das matrizes foi feito ao longo do ano de 2011, com observações mensais, anotadas em planilhas de campo e utilizando-se uma câmera fotográfica. Foi considerada a presença ou ausência da fenofase, não tendo sido quantificada sua intensidade. As fenofases consideradas no estudo foram: estado vegetativo, floração, frutificação (frutos maduros e frutos dispersando).

Foi calculado o índice de sincronismo para a floração e frutificação de matrizes da mesma espécie através da fórmula do índice de sincronia $(Z)$ adaptado de Augspurger (1983):

$$
Z=\sum_{I=1}^{n} X_{1} / n
$$

onde, $\mathrm{X}_{\mathrm{i}}=$ sincronia do indivíduo i com seus coespecíficos $; \mathrm{n}=$ número de indivíduos na população. 
Calculou-se $X_{i}$ pela seguinte fórmula:

$$
X_{i}=(1 / n-1) \cdot\left(1 / f_{i}\right) \cdot \sum_{i=1}^{n} e_{j \neq i}
$$

onde, $\mathrm{e}_{\mathrm{j}}=$ número de registros nos quais ambos os indivíduos i e $\mathrm{j}$ estão na mesma fenofase; $\mathrm{f}_{\mathrm{i}}=$ número de registros nos quais o indivíduo i está na fenofase considerada.

A influência dos fatores climáticos na floração e na frutificação foi investigada através do coeficiente de correlação de Spearman, correlacionando a ocorrência das fenofases e as variáveis climáticas de cada mês (precipitação total, temperaturas médias mensais e fotoperíodo). A mesma análise foi repetida entre as variáveis climáticas e a ocorrência da frutificação, analisando separadamente as espécies com frutos zoocóricos e não zoocóricos.

Para o fotoperíodo considerou-se a média histórica de 1963 a 1990 dos valores de insolação total mensal da Estação Meteorológica do Rio de Janeiro do INMET, devido à impossibilidade de obtenção desta informação junto a Estação Meteorológica de Sambaetiba (Itaboraí, RJ). Os demais dados climáticos foram obtidos para o ano de 2011, na Estação de Sambaetiba, Itaboraí, RJ.

\section{Resultados e discussão}

Os 91 indivíduos estudados eram pertencentes a 9 famílias, sendo a maioria de Fabaceae $(n=7)$. Das 91 matrizes marcadas, foi observada floração em $85 \%$ e frutificação em $87 \%(\mathrm{n}=79)$. As demais permaneceram em estado vegetativo, ou não foi possível visualizar o episódio de floração, como foi caso das espécies Dalbergia nigra e Machaerium brasiliense. Isso ocorreu provavelmente devido à baixa frequência de idas ao campo (uma vez por mês) e à rápida floração destas espécies. Estas mesmas espécies frutificaram posteriormente indicando que já se encontram em idade reprodutiva. Na tabela 1 é apresentado o calendário fenológico de floração e frutificação das 21 espécies, sendo sete espécies pioneiras, sete secundárias iniciais e sete secundárias tardias.

O maior número de espécies em floração foi observado nos meses de setembro e novembro de 2011 (Figura 2). Este período corresponde ao final da estação seca e início da chuvosa. Comunidades vegetais sujeitas a climas sazonais, que possuem estações seca e úmida bem marcadas, apresentam período de floração maior na transição da estação seca para a estação úmida, ou na estação seca (van Schaik et al., 1993; Justiniano \& Fredericksen, 2000; Ochoa-Gaona., et al., 2008; Gomes et al., 2008). Esse comportamento tem sido atribuído à redução do estresse hídrico causado pelas primeiras chuvas após o período de seca, ao aumento do fotoperíodo e à elevação da temperatura (Morellato, 1995). Além disso, a estação chuvosa pode acarretar o aumento dos danos mecânicos às inflorescências, sendo a estação seca o período com maior abundância de polinizadores (van Schaik et al., 1993; Fisch et al., 2000). Entretanto, neste estudo só foi observada correlação significativa entre floração e fotoperíodo, sendo esta relação moderada e negativa (Tabela 2 ). $\mathrm{O}$ fotoperíodo e a temperatura são apontados como fatores indutores do florescimento, sobretudo em climas menos sazonais, sendo esperada uma correlação positiva entre eles o período de floração e os dias mais longos ou temperaturas mais elevadas (Alvin \& Alvin, 1976; Talora \& Morellato, 2000; Morellato et al., 2000; van Schaik et al., 1993).

O maior número de espécies frutificando foi observado de março a abril e de agosto a setembro (Figura 3; Tabela 1). Liebsch \& Mikich (2009) estudando a fenologia de 543 indivíduos de 145 espécies em Floresta Ombrófila Densa no Paraná observaram que a frutificação concentrou-se entre dezembro e abril, com pico de atividade em fevereiro, e que nos meses mais frios (junho a agosto), foi encontrado o menor número de espécies em estado fértil (flor ou fruto).

As espécies com dispersão zoocórica (48\%) apresentaram pico de frutificação em março e abril, período de alta precipitação, enquanto as espécies anemocóricas e/ou autocóricas tiveram pico de frutificação em agosto a setembro, período de menor precipitação. A análise da correlação de Spearman mostrou que houve correlação entre precipitação e frutificação de espécies zoocóricas (Tabela 2).

As espécies zoocóricas possuem frutos carnosos, e a disponibilidade de água parece ser uma necessidade importante para a frutificação (Alencar, 1996). Alguns autores defendem que neste período a disponibilidade de dispersores é maior (Rathcke \& Lacey, 1985; Morellato \& Leitão-Filho, 1992). O mesmo padrão foi encontrado para espécies de Sapotaceae, com frutos carnosos, na Amazônia, por Alencar (1996) e vem sendo relatado em vários outros estudos (Wikander, 1984; Locatelli \& Machado, 2004; Piña-Rodrigues et al., 2007). 
Tabela 1. Época de floração e frutificação de 21 espécies arbóreas em Itaboraí, RJ.

\begin{tabular}{|c|c|c|c|c|c|c|c|}
\hline Família/ Espécie & $\mathbf{n}$ & GE & $\begin{array}{l}\text { Síndrome de } \\
\text { dispersão }\end{array}$ & Floração & $\mathbf{Z}_{\text {flor }}$ & Frutificação & $\mathbf{Z}_{\text {frut }}$ \\
\hline \multicolumn{8}{|l|}{ Cannabaceae } \\
\hline Trema micrantha (L.) Blume & 5 & $\mathrm{P}$ & Zoo & Mar-dez & 0,32 & nov-mar & 0,37 \\
\hline \multicolumn{8}{|l|}{ Fabaceae } \\
\hline Albizia pedicellaris (DC.) L. Rico & 7 & $\mathrm{P}$ & Auto & jan-mar; nov & 0,07 & jan-fev; ago-set & 0,07 \\
\hline Andira sp. & 4 & SI & Zoo & set & 0,25 & abr-mai & 0,25 \\
\hline Apuleia leiocarpa (Vog.) J. F. Macbr & 5 & $\mathrm{ST}$ & Ane & ago-set & 0,05 & fev-abr & 0,35 \\
\hline Dalbergia foliolosa Benth. & 6 & ST & Ane & fev & 0,60 & ago-set & 0,30 \\
\hline Dalbergia nigra (Vell.) Allemão ex. Benth. & 3 & SI & Ane & $*$ & - & ago-set & 0,67 \\
\hline Enterolobium glaziovii (Benth.) Mesquita & 5 & SI & Auto & set-out & 0,60 & jun-set & 0,30 \\
\hline Inga vera subsp. affinis (DC.) T. D. Penn. & 2 & SI & Zoo & ago-nov & 0,00 & jan-fev & 0,50 \\
\hline Machaerium brasiliense Vogel & 2 & ST & Ane & $*$ & - & jun;set & 0,0 \\
\hline Machaerium hirtum (Vell.) Stellfeld & 11 & $\mathrm{P}$ & Ane & jan-mar & 0,54 & ago-set & 0,45 \\
\hline Melanoxylon brauna Schott & 3 & $\mathrm{ST}$ & Ane & jun;dez & 0,17 & mai-jul & 0,17 \\
\hline Mimosa bimucronata (DC.) Kuntze & 4 & $\mathrm{P}$ & Auto & jul-ago & 0,50 & abr-mai & 0,25 \\
\hline Stryphnodendron polyphyllum var. villosum Benth. & 4 & SI & Auto & set-dez & 0,16 & mar-mai & 0,22 \\
\hline Swartzia oblata R. S. Cowan & 2 & $\mathrm{ST}$ & Zoo & mar & 0,5 & ago & 0,5 \\
\hline \multicolumn{8}{|l|}{ Lamiaceae } \\
\hline Aegiphila sellowiana Cham. & 5 & $P$ & Zoo & jan; jun-dez & 0,12 & fev-abr; jul-set & 0,08 \\
\hline \multicolumn{8}{|l|}{ Malpiguiaceae } \\
\hline Byrsonima sericea DC. & 3 & SI & Zoo & nov-dez & 0,33 & mar-mai & 0,67 \\
\hline \multicolumn{8}{|l|}{ Malvaceae } \\
\hline Luehea grandiflora Mart. \& Zucc. & 6 & $\mathrm{P}$ & Ane & mar-ago & 0,53 & ago-set & 0,30 \\
\hline \multicolumn{8}{|l|}{ Meliaceae } \\
\hline Guarea guidonia (L.) Sleumer & 3 & $\mathrm{ST}$ & Zoo & nov-dez & 0,50 & fev-abr & 0 \\
\hline \multicolumn{8}{|l|}{ Myrtaceae } \\
\hline Psidium guineense Sw. & 2 & $\mathrm{P}$ & Zoo & out-nov & 0,17 & mar & 0,17 \\
\hline \multicolumn{8}{|l|}{ Rubiaceae } \\
\hline Genipa americana $\mathrm{L}$. & 3 & SI & Zoo & nov & 0 & fev-out & 0 \\
\hline \multicolumn{8}{|l|}{ Sapindaceae } \\
\hline Cupania racemosa (Vell.) Radlk. & 6 & $\mathrm{ST}$ & Zoo & abr-mai & 0,83 & out-dez & 0,50 \\
\hline
\end{tabular}

Número de matrizes avaliadas (n); síndrome de dispersão - zoo = zoocórica; auto = autocórica; ane = anemocórica; GE = grupo ecológico por espécie $\left(\mathrm{P}=\right.$ pioneira; $\mathrm{SI}=$ secundária inicial; $\mathrm{ST}$ = secundária tardia); $\mathrm{Z}_{\mathrm{flor}}=$ índice de sincronismo da floração; $\mathrm{e} \mathrm{Z}_{\text {frut }}=$ índice da frutificação entre matrizes da mesma espécie. * Casos em que não foi observada a floração.

Ao contrário, espécies de leguminosas cujos frutos são secos necessitam que seus frutos sofram desidratação do pericarpo, para ocorrer sua deiscência (Mantovani \& Martins, 1988), e por isso frutificam na estação seca. Reys et al. (2005) estudando 29 espécies em mata ciliar em Bonito, MS, observou que a maior parte das espécies anemocóricas frutificou nos meses de julho e agosto (sendo julho o mês mais seco do ano de estudo).
A maioria das espécies (81\%) floresceu uma vez ao longo de 2011. Apenas duas espécies (9,5\%) floresceram mais de uma vez no ano analisado (Apuleia leiocarpa e Trema micrantha). Em relação à frutificação, as espécies Aegiphila sellowiana, Albizia pedicellaris, Genipa americana eTrema micrantha frutificaram duas ou mais vezes por ano, enquanto as demais espécies frutificaram uma única vez. Entretanto, mais tempo de estudo é necessário para avaliar o comportamento 
reprodutivo, identificar os padrões de floração (contínua, anual, sub-anual ou supra-anual), ou mesmo aferir sobre a regularidade destes eventos (Newstrom et al., 1994).

$\mathrm{O}$ índice de sincronismo de floração variou de 0 a 0,83 , tendo sido abaixo de 0,60 para a maioria das espécies (Tabela 1). As espécies que apresentaram maior valor foram Cupania racemosa $(\mathrm{z}=0,83)$, Enterolobium glaziovii (z=0,60) e Dalbergia foliolosa $(\mathrm{z}=0,60)$. As espécies com menor valor de sincronismo foram Genipa americana e Inga vera subsp. affinis, que alcançaram valor zero. Seguindo o padrão da floração, a frutificação também apresentou baixos valores de sincronismo, variando de zero, para Guarea guidonia e Genipa americana, a 0,67, para Dalbergia nigra e Byrsonima sericea. As espécies Albizia pedicellaris e Aegiphila sellowiana apresentaram valores bem baixos de sincronismo, com 0,07 e 0,08 , respectivamente (Tabela 1). A alta sincronia de Byrsonima sericea já foi observada por Teixeira \& Machado (2000), em Recife, PE, e pode ser explicada evolutivamente pela necessidade da espécie em realizar polinização cruzada (xenogamia), uma vez que é auto-incompatível.

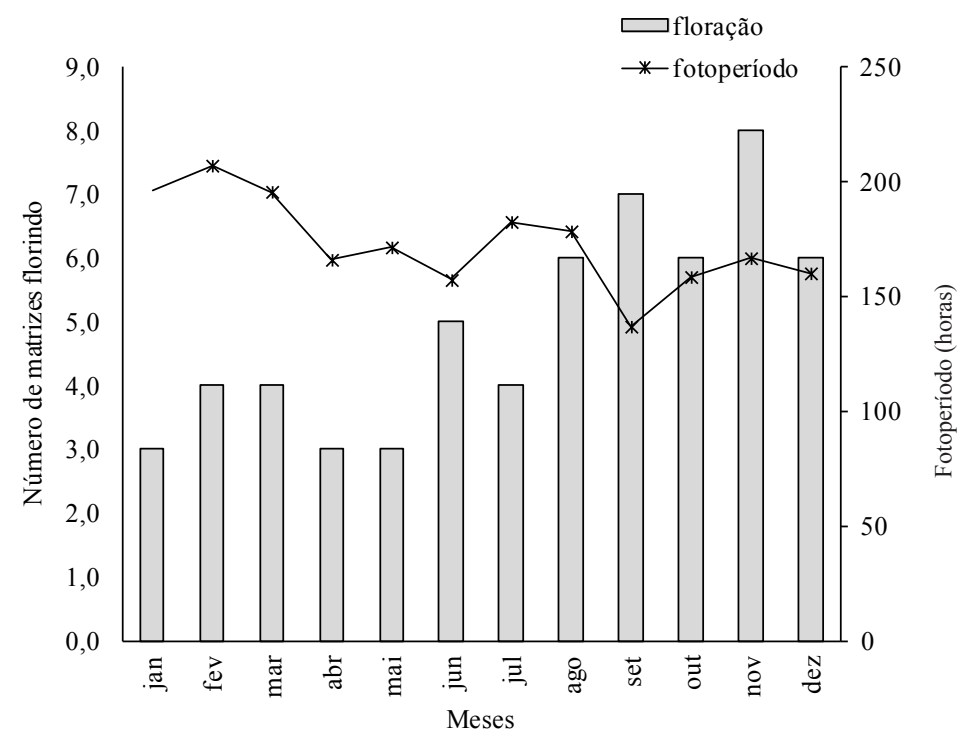

Figura 2. Número total de espécies em floração e fotoperíodo no período de janeiro a dezembro de 2011, em Itaboraí, RJ.

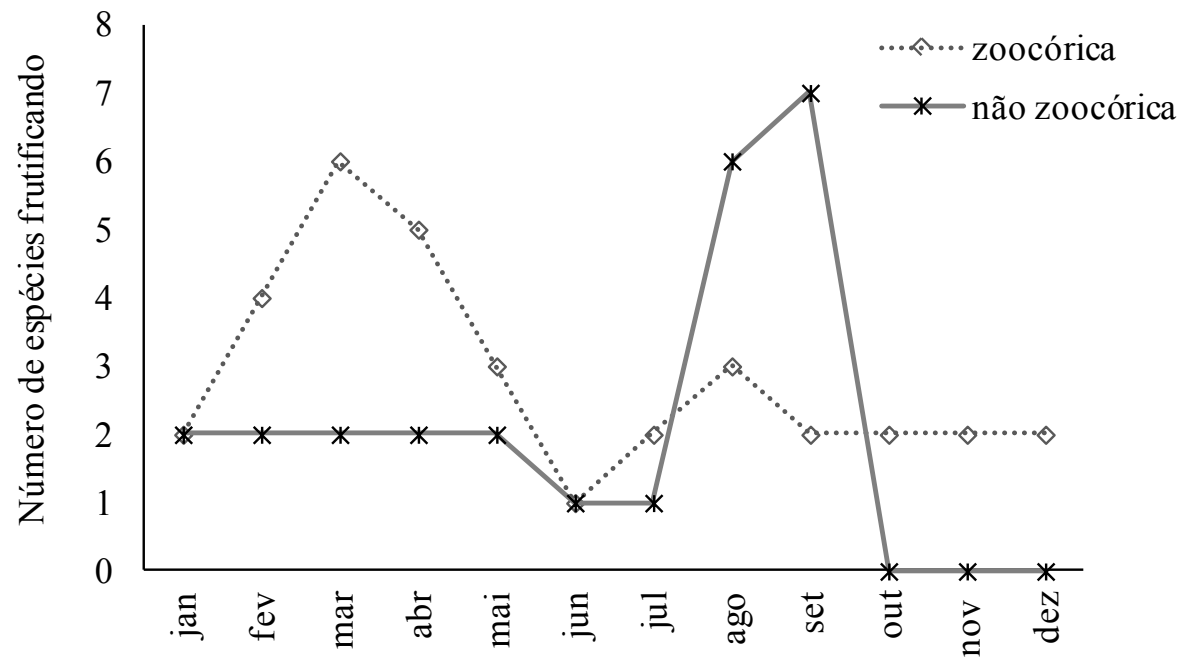

Figura 3. Número de espécies em frutificação no período de janeiro a dezembro de 2011, em Itaboraí, RJ. 
Tabela 2. Valores da análise de correlação de Spearman (rs) entre as variáveis climáticas (precipitação, temperaturas médias mensais e fotoperíodo) e as fenofases reprodutivas (floração e frutificação) de 21 espécies arbóreas em Itaboraí, RJ.

\begin{tabular}{lcccc}
\hline & Flor & Fruto & Fruto zoo & $\begin{array}{c}\text { Fruto } \\
\text { não zoo }\end{array}$ \\
\hline Temperatura & $-0,2452$ & $-0,0560$ & 0,4304 & $-0,1975$ \\
Precipitação & 0,1261 & $-0,1366$ & $0,5573^{*}$ & $-0,3210$ \\
Fotoperíodo & $-0,5784^{*}$ & 0,1507 & 0,1735 & 0,1991 \\
\hline
\end{tabular}

flor = floração de todas as espécies; fruto = frutificação de todas as espécies; fruto zoo = frutificação das espécies zoocóricas; fruto não zoo = frutificação das espécies não zoocóricas. *Significativo a $\mathrm{P}<0,05$.

A maioria das matrizes estudadas estão localizadas em bordas de fragmentos, em uma paisagem intensamente fragmentada, muitas vezes isoladas, não constituindo populações contínuas. Existem poucos trabalhos publicados sobre o efeito do isolamento e da fragmentação sobre a fenologia de espécies arbóreas, e os resultados obtidos são controversos. Alguns estudos tem indicado que algumas espécies se beneficiam com a fragmentação, aumentando o sincronismo, a duração da floração e a produção de sementes e frutos (Andreazzi et al., 2012; Athaíde, 2007; Herrerías-Diego et al., 2006; Fuchs et al., 2003). Já outros estudos indicam que flores de árvores isoladas recebem menos pólen e produzem menos frutos do que árvores situadas em florestas (Rocha \& Aguilar, 2001; Aizen \& Feinsinger, 1994). A continuidade do estudo de fenologia por mais anos, a ampliação do número de matrizes estudadas por espécie e a realização de estudos complementares de biologia floral são fundamentais para avaliar os reais mecanismos que podem estar interferindo na produção de sementes e no baixo sincronismo das matrizes estudadas em Itaboraí.

As sementes de algumas espécies apresentaram-se comprometidas pela incidência de pragas. Foi o caso do Machaerium hirtum, Melanoxylon brauna, Machaerium brasiliense e Psidium guineense, que perderam lotes inteiros devido a pragas. Outras espécies apresentaram baixa produção de frutos, como foi o caso de Andira sp. Swartzia oblata, Guarea guidonea e Enterolobium glaziovii. Talvez o fato destas matrizes estarem isoladas em virtude do intenso processo de fragmentação da região, tenha dificultado a chegada de polinizadores e consequentemente a ocorrência de fecundação. Mais de $80 \%$ das angiospermas dependem de animais para sua polinização e reprodução sexual (Buchmann \& Nabhan, 1996; Bawa, 1990), de maneira que a dependência dessas relações mutualísticas para reprodução pode ampliar a suscetibilidade da planta à fragmentação ou outras formas de perturbação do ambiente. Pinheiro \& Sazima (2007) indicaram que a polinização cruzada é obrigatória para formação de frutos para Swartzia oblata, sendo seu polinizador a abelha grande. A espécie Guarea guidonea possui flores dióicas (Styles, 1972) e síndrome de polinização cantarofilia (borboletas) (Kinoshita et al., 2006). Para Enterolobium glaziovii não existem estudos já publicados sobre biologia floral, entretanto já foi observado que sua congênere, E. cyclocarpum, é predominantemente alógama e com suspeitas de autoincompatibilidade (Rocha \& Aguilar, 2001).

Em relação ao período de floração e frutificação foi observado que sete espécies (33\%) tiveram período reprodutivo diferente do relatado na bibliografia para outras regiões. Não foi encontrada publicação de fenologia para quatro das 21 espécies avaliadas (Cupania racemosa, Dalbergia foliolosa, Machaerium brasiliense e Albizia pedicellaris). As seguintes espécies coincidiram total ou parcialmente o período de floração e frutificação com outras referências bibliográficas: Aegiphila sellowiana (Pereira et al., 2008; Liebsch \& Mikich, 2009), Apuleia leiocarpa (Pereira et al., 2008), Byrsonima sericea (Braga et al., 2010; Locatelli \& Machado, 2004; Dunley, 2006), Inga vera subsp. affinis (Lopes et al., 1996; Brandão et al., 2002), Machaerium hirtum (Sartori \& Tozzi, 1998), Melanoxylon brauna (Ramalho \& Marangon, 1989), Mimosa bimucronata (Carvalho, 2003), Stryphnodendron polyphyllum var. villosum (Pereira et al., 2008) e Trema micrantha (Pereira \& Amaral, 2008). Estas informações reforçam a necessidade de realização de estudos fenológicos em regiões bioclimáticas específicas como forma de subsidiar ações de colheita de sementes e recuperação ambiental.

\section{Conclusões}

O período com maior quantidade de espécies frutificando foi de março a abril (espécies zoocóricas) e de agosto a setembro (espécies anemocóricas).

Houve correlação significativa entre a precipitação e a produção de frutos carnosos dispersos por animais (zoocóricos). 
A floração apresentou correlação negativa com o fotoperíodo.

Houve baixa sincronia nos episódios de floração e frutificação entre as matrizes para a maioria das espécies estudadas.

Cerca de 1/3 das espécies estudadas não forneceu sementes em quantidade ou qualidade satisfatória para a coleta, seja devido à baixa produção de frutos ou à incidência de pragas ou doenças.

A baixa disponibilidade de pesquisas sobre fenologia para grande parte das espécies estudadas, reforça a necessidade de se realizar mais estudos sobre este tema, em regiões bioclimáticas específicas.

\section{Agradecimentos}

À Janaina Ribeiro, pelo auxílio nas análises estatísticas, à Cetrel-Lumina Tecnologia e Engenharia Ambiental Ltda., pelos dados meteorológicos, ao Fernando Lima, pelo apoio no trabalho de campo, e ao Luiz Fernando Duarte de Moraes, pela revisão do trabalho.

\section{Referências}

AIZEN, M. A.; FEINSINGER, P. Forest fragmentation, pollination, and plant reproduction in a chaco dry forest, Argentina. Ecology, New York, v. 75, p. 330-351, 1994.

ALENCAR, J. da C. Fenologia de cinco espécies arbóreas tropicais de sapotaceae correlacionada a variáveis climáticas na Reserva Ducke, Manaus, AM. Acta Amazonica, Manaus, v. 24, n. 3/4, p. 161-182, 1996.

ALVIN, P. T.; ALVIN, R. Relation of climate to growth periodicity in tropical trees. In: TOMLINSON, P. B.; ZIMMERMANN, M. H. (Ed.). Tropical trees as living systems. London: Cambridge University Press, 1976. p. 445-464.

ANDREAZZI, C. S.; PIMENTAL, C. S.; PIRES,A. S.; FERNANDEZ, F. A. S.; OLIVEIRA-SANTOS, L. G.; MENEZES, J. F. S. Increased productivity and reduced seed predation favor a large-seeded palm in small Atlantic Forest Fragments. Biotropica, Washington, US, v. 44, n. 2, p. 237-245, 2012.

ATHAYDE, E. A. Influência da borda e do isolamento na fenologia e no sucesso reprodutivo de Anadenanthera falcata (BENTH.) Speg. (Fabaceae) em uma região de cerrado stricto sensu, Itirapina, São Paulo. 2007. 48 f. Tese (Mestrado em Biologia Vegetal) - Universidade Estadual Paulista, São Paulo.

AUGSPURGER, C.K. Phenology, flowering synchrony and fruit set of six neotropical shrubs. Biotropica, v.15, p.257-267,1983.

BAWA, K. S. Plant-pollinatior interactions in tropical rain forests. Annual Review of Ecology and Systematics, Boston, v. 21, p. 399-422, 1990.
BENCKE, C. S. C.; MORELLATO, L. P. C. Estudo comparativo da fenologia de nove espécies arbóreas em três tipos de floresta atlântica no sudeste do Brasil. Revista Brasileira de Botânica, São Paulo, SP, v. 25, p. 237-248, 2002.

BONNET, A.; RESENDE, A. S. de; CURCIO, G. R. (Org.). Manual de plantio de espécies nativas para o corredor ecológico do Comperj. Seropédica: Embrapa Agrobiologia; Colombo: Embrapa Florestas; Rio de Janeiro: Embrapa Solos, 2009. 155 p.

BRAGA, L. O.; CUNHA, E.J.R.da; SILVA, A.M. de S.; OLIVEIRA, M.da C.P. de. Estudos fenológicos de quatro espécies de plantas de cerrado de interesse ecológico e econômico ocorrentes no Eco Resort Nazareth, município de José de Freitas. In: SEMINÁRIO DE INICIAÇÃO CIENTÍFICA, 19.; SEMINÁRIO EM DESENVOLVIMENTO TECNOLÓGICO E INOVAÇÃO, 2., 2010, Teresina. Anais eletrônicos...]. Teresina: UFP, 2010. Disponível em: <http:/www.ufpi.br/19sic/Documentos/RESUMOS/ Vida/Lucas\%20Oliveira\%20Braga.pdf $>$. Acessoem: 02 out.2012

BRANDÃO, M.; LACA-BUENDIA, J. P.; MACEDO, J. F. Árvores nativas e exóticas do Estado de Minas Gerais. Belo Horizonte: EPAMIG, 2002. 528 p.

BUCHMANN, S. L.; NABHAN, G. P. The forgotten pollinators. California: Island Press, Covelo, 1996.

CARVALHO, P. E. R. Espécies arbóreas brasileiras. Brasília, DF: Embrapa Informação Tecnológica; Colombo: Embrapa Florestas, 2003. 1039 p. v. 1.

CARVALHO, P. E. R. Espécies arbóreas brasileiras. Brasília, DF: Embrapa Informação Tecnológica; Colombo: Embrapa Florestas, 2006. 628 p. v. 2.

CARVAlHO, F. A.; NASCIMENTO, M. T.; BRAGA, J. M. A. Estrutura e composição florística do estrato arbóreo de um remanescente de mata atlântica submontana no município de Rio Bonito, RJ, Brasil (Mata Rio Vermelho). Revista Brasileira de Biociências, Porto Alegre, v. 5, suplemento 1, p. 423-425, 2007.

DUNLEY, B. S. Biologia reprodutiva de Byrsonima sericea (Malpiguiaceae), em fragmentos de diferentes tamanhos na Restinga de Massambaba, Arraial do Cabo, Rio de Janeiro. 2006. 52 f. Dissertação (Mestrado em Botânica) - Escola Nacional de Botânica Tropica, Instituto de Pesquisas do Jardim Botânico do Rio de Janeiro, Rio de Janeiro.

FISCH, S. T. V.; NOGUEIRA JUNIOR, L. R.; MANTOVANI, W. Fenologia reprodutiva do Euterpe edulis Mart. na Reserva Ecológica do Trabiju (Pindamonhangaba - SP). Revista Biociências, São Paulo, v. 6, n. 2, p. 31-37, 2000.

FOURNIER, L. A. El dendrofenograma, una representación gráfica del comportamiento de losárbores. Turrialba, Turrialba, v. 26, n. 1, p. 96-97, 1976.

FOURNIER, L. A. Un método cuantitativo para la medición de características fenológicas em árbores. Turrialba, Turrialba, v. 24, n. 4, p. 422-423, 1974.

FUCHS, E. J.; LOBO, J. A.; QUESADA, M. Effects of forest fragmentation and flowering phenology on the reproductive success and mating patterns of the dry forest tree Pachira quinata. Conservation Biology, Boston, v. 17,n. 1, p.149-157, 2003. 
GANDOLFI, S.; LEITÃO FILHO, H.; BEZERRA, C. L. F. Levantamento florístico e caráter sucessional das espécies arbustivoarbóreas de uma floresta mesófila semidecídua no município de Guarulhos-SP. Revista Brasileira de Botânica, São Paulo, SP, v. 55, n. 4, p. 753-767, 1995.

GOMES, R.; PINHEIRO, M. C. B.; LIMA, H. A.de. Fenologia reprodutiva de quatro espécies de Sapotaceae na restinga de Maricá, RJ. Revista Brasileira de Botânica, São Paulo, SP, v. 31, n. 4, p. 679-687, out./dez, 2008

HERRERÍAS-DIEGO, Y.; QUESADA, M.; STONER, K. E.; LOBO, J. A. Effects of forest fragmentation on phenological patterns and reproductive success of the tropical dry forest tree Ceiba aesculifolia. Conservation Biology, Boston, v. 20, p. 1111-1120, 2006.

KAGEYAMA, P.; GANDARA, F. B. Recuperação de áreas ciliares. In: Rodrigues, R. R.; LEITÃO FILHO, H. F. (Ed.). Matas ciliares: conservação e recuperação. São Paulo: EDUSP, 2000. p. 249-269.

KINOSHITA, L. S.; TORRES, R. B.; FORNI-MARTINS, E. R.; SPINELlI, T.; AHN, Y. J.; CONSTÂNCIO, S. S. Composição florística e síndromes de polinização e de dispersão da mata do Sítio São Francisco, Campinas, SP, Brasil. Acta Botanica Brasilica, Porto Alegre, v. 20, n. 2, p. 313-327, 2006.

KÖPPEN, W. Climatologia: com um estúdio de los climas de la tierra. New Gersey: Climatology,1948. 104p.

JUSTINIANO, M. J.; FREDERICKSEN, T. S. Phenology of tree species in Bolivian dry forests. Biotropica, Washington, US, v. 32, p. $276-281,2000$.

LIEBSCH, D.; MIKICH, S. B. Fenologia reprodutiva de espécies vegetais da Floresta Ombrófila Mista do Paraná, Brasil. Revista Brasileira Botânica, São Paulo, SP, v. 32, n. 2, p. 375-391, 2009.

LIMA, C. L. R.; SILVA, A. P.; IMHOFF, S.; LEÃO, T. P. Estimativa da capacidade de suporte de carga do solo a partir da avaliação da resistência a penetração. Revista Brasileira de Ciência do Solo, Viçosa, MG, v. 30, n. 2, p. 217-223, mar/abr, 2006.

LOCATELLI, E.; MACHADO, I. C. Fenologia d as Espécies Arbóreas de uma Mata Serrana (Brejo de Altitude) em Pernambuco, Nordeste do Brasil. In: PORTO, K. C.; CABRAL, J. J. P.; TABARELLI, M. (Org.). Brejos de altitude em Pernambuco e Paraíba: história natural, ecologia e conservação. Brasília, DF: Ministério do Meio Ambiente, 2004. p. 255-276. (Série Biodiversidade, 9).

LOPES, B.C.; FERREIRA, M. B. D.; BRANDÃO, M. Sombreamento em pastagens: espécies recomendadas para as diversas regiões do estado de Minas Gerais. Daphne, Belo Horizonte, v. 6, n. 4, p. 7-15, out. 1996.

MANTOVANI, W.; MARTINS, F. R. Variações fenológicas das espécies do cerrado da Reserva Biológica de Moji Guaçu, Estado de São Paulo. Revista Brasileira de Botânica, São Paulo, v. 11 n. 1/2, p. 101-112, 1988.

MANTOVANiI, M.; RUSCHEL, A. R.; REIS, M. S. dos; PUCHALSKI, A.; NODARI, R. O. Fenologia reprodutiva de espécies arbóreas em uma formação secundária da Floresta Atlântica. Revista Árvore, Viçosa, MG, v. 27, n. 4, p. 451-458, 2003.
MARIOT, A.; MANTOVANI, A.; REIS, M. S. Uso e conservação de Piper cernuum Vell. (Piperaceae) na Mata Atlântica: I. fenologia reprodutiva e dispersão de sementes. Revista Brasileira de Plantas Medicinais, Botucatu, v. 5, n. 2, p. 1-10, 2003.

MEDEIROS, A. C. de S.; NOGUEIRA, A. C. Planejamento da coleta de sementes florestais nativas. Colombo: Embrapa Florestas, 2006. 9 p. (Embrapa Florestas. Circular técnica, 126).

MIJNSBRUGGE. C. V.; BISCHOFF, A.; SMITH, B. A question of origin: where and how to colletc seed for ecological restoration. Basic and Applied Ecology, Gottingen, v. 11, p. 300-311, 2010.

MOREIRA, P. R.; ANANIA, P. F. Proposta técnica para identificação de árvores matrizes, coleta de sementes e produção de mudas de árvores nativas da flora regional. Limeira, jul, 2005. Disponível em: <http://www.floresta.eng.br/trabalhos\%20tecnicos/ Proposta\%20Tecnica.pdf>. Acesso em: 10 maio 2011.

MORELlATO, L. P. C. As estações do ano na floresta. In: MORELlATO, P. C.; LEITÃO FILHO, H. F. (Org.). Ecologia e preservação de uma floresta tropical urbana. Campinas: Unicamp, 1995. p. 37-41.

MOREllato, L. P. C.; LEITÃo FILHO, H. F. Padrões de frutificação e dispersão na Serra do Japi. In: MORELLATO, L. P. C. (Ed.). História natural da Serra do Japi: ecologia e preservação de uma área florestal no sudeste do Brasil. Campinas: FAPESP, 1992. p. 112-140.

MORELlATO, L. P. C.; TALORA, D. C.; TAKAHASI, A.; BENCKE, C. C.; ROMERA, E. C.; ZIPPARRO, V. B. Phenology of Atlantic rain forest trees: a comparative study. Biotropica, Washington, US, v. 32, p. 811-823, 2000.

NEWSTROM, L. E.; FRANKIE, G. W.; BAKER, H. G. A new classification for plant phenology based on flowering in lowland tropical rain forest trees at La Selva, Costa Rica. Biotropica, Washington, US, v. 2, p. 141-159, 1994.

OCHOA-GAONA, S.; HERNÁNDEZ, I. P.; JONG, B. H. J. de. Fenología reproductiva de las especies arbóreas del bosque tropical de Tenosique, Tabasco, México. Revista de Biologia Tropical, San Jose, v. 56, n. 2, p. 657-673, 2008.

PEREIRA, S.G.; AMARAL, A.F. Fenologia das espécies arbóreas do bosque do Parque Municipal do Mocambo, Patos de Minas - MG. Perquirēre:Revista Eletrônica da Pesquisa, edição 5, ano 5, jun. 2008.

PEREIRA, T. S.; COSTA, M. L. M. N. da; MORAES, L. F. D.; LUCHIARI, C. Fenologia de espécies arbóreas em floresta Atlântica da Reserva Biológica de Poço das Antas, Rio de Janeiro, Brasil. Iheringia, Série Botânica, Porto Alegre, v. 63, n. 2, p. 329-339, jul./dez. 2008.

PIÑA-RODRIGUES, F. C. M.; FREIRE, J. M.; LELES, P. S. dos S.; BREIER, B. T. Parâmetros técnicos para produção de sementes florestais: Rede Mata Atlântica de sementes florestais. Seropédica: EDUR, 2007. 188 p.

PINHEIRO, M.; SAZIMA, M. visitantes florais e polinizadores de seis espécies arbóreas de Leguminosae Melitófilas na Mata Atlântica no Sudeste do Brasil. Revista Brasileira de Biociências, Porto Alegre, v. 5, suplemento 1, p. 447-449, jul. 2007. 
PIRES, A. C. B. A. Conservação genética de espécies arbóreas nativas em remanescentes de Mata Atlântica no município do Rio de Janeiro. 2008. 31 f. Monografia (Graduação em Engenharia Florestal) - Universidade Federal Rural do Rio de Janeiro, Seropédica, RJ.

RAMALHO, R. S.; MARANGON, L. C. Características fenológicas de Melanoxylon brauna Schott., em Viçosa-Minas Gerais. Revista Arvore, Viçosa, MG, v. 13, n. 2, p. 203-209, 1989.

RATHCKE, B.; LACEY, E. P. Phenological patterns of terrestrial plants. Annual Review of Ecology and Systematics, Palo Alto, v. 16, p. 179-214, 1985.

REYS, P.; GALETTI, M.; MORELlATO, L. P. C.; SABINO, J. Fenologia reprodutiva e disponibilidade de frutos de espécies arbóreas em mata ciliar no rio Formoso, Mato Grosso do Sul. Biota Neotropica, Campinas, v.5, n.2, p. 309-318, 2005. RIO DE JANEIRO (Estado). Secretaria de Estado do Ambiente.Instituto Estadual do Ambiente. Diagnóstico da produção de mudas de espécies nativas no Estado do Rio de Janeiro. Rio de Janeiro,2010. 96 p. Disponível em: <http://download.rj.gov.br/documentos/10112/390027/DLFE25238.pdf/diagnostico_mudas_VF.pdf >. Acesso em: 15 maio 2011.

RIO DE JANEIRO (Estado). Secretaria de Estado do Ambiente. Instituto Estadual do Ambiente. Diagnóstico da produção de mudas de espécies nativas no Estado do Rio de Janeiro. Rio de Janeiro, 2010. 96 p. Disponível em: <http://download.rj.gov.br/ documentos/10112/390027/DLFE-25238.pdf/diagnostico_mudas_ VF.pdf $>$. Acesso em: 15 maio 2011.

ROCHA, O. C.; AGUILAR, G. Reproductive biology of the dry Forest tree Enterolobium cyclocarpum (guanacaste) in Costa Rica: a comparison between trees left in pastures and trees in continuous forest. American Journal of Botany, Columbus, v. 88, n. 9, p. 1607-1614, 2001.
SARTORI, A. B.; TOZZI, A. M. G. A. As espécies de Machaerium Pers. (Leguminosae - Papilionoideae - Dalbergieae) ocorrentes no Estado de São Paulo. Revista Brasileira de Botânica, São Paulo, v. 21, n. 3, dec. 1998.

SEBBENN, A. M. Número de árvores matrizes e conceitos genéticos na coleta de sementes para reflorestamentos com espécies nativas. Revista do Instituto Florestal, São Paulo, SP, v. 14, p.115-132, 2002.

SHIMIZU. J. Y. Estratégia complementar para conservação de espécies florestais nativas: resgate e conservação de ecótipos ameaçados. Pesquisa Florestal Brasileira, Colombo, n. 54, p. 7-35, jan./jun. 2007.

STYLES, B. T. The flower biology of the Meliaceae and its beraring on tree breeding. Silvae Genetica, Frankfurt, v. 21, n.5, p. 149-204, 1972.

TAlORA, D. C.; MORELlATO, P. C. Fenologia de espécies arbóreas em floresta de planície litorânea do sudeste do Brasil. Revista Brasileira de Botânica, São Paulo, v. 23, p. 13-26. 2000.

TEIXEIRA, L. A. G.; MACHADO, I. C. Sistema de polinização e reprodução de Byrsonima sericea DC. (Malpighiaceae). Acta Botanica Brasilica, Porto Alegre, v. 14, p. 347-357, 2000.

TONETTO, T. da S.; PRADO, A. P. do; ARAUJO, M. M.; SCCOTI, M. S. V.; FRANCO, E. T. H. Dinâmica populacional e produção de sementes de Eugenia involucrata na Floresta Estacional Subtropical. Floresta e Ambiente, Seropédica, v. 20, n. 1, p. 62-69, 2013.

VAN SCHAIK, C. P.; TERBORGH, J. W.; WRIGHT, S. J. The phenology of tropical forests: adaptive significance and consequences for primary consumers. Annual Review of Ecology and Systematics, Palo Alto, v. 24, p. 353-377, 1993.

WIKANDER, T. Mecanismos de dispersión de diasporas de una selva decidua en Venezuela. Biotropica, Washington, US, v. 16, p. 276-283, 1984. 